Alimentary tract

\title{
Ustekinumab in bio-naïve and bio-failure Crohn's disease patients: Results from a " real-life » monocentric cohort.
}

\author{
L. Monin ${ }^{\text {a }}$, S. Dubois ${ }^{a}$, C. Reenaers ${ }^{\text {a }}$, C. Van Kemseke ${ }^{a}$, P. Latour ${ }^{a}$, D. Van Daele ${ }^{a}$, \\ S Vieujean ${ }^{\mathrm{a}}$, L. Seidel ${ }^{\mathrm{b}}$, E. Louis ${ }^{\mathrm{a}, *}$
}

${ }^{a}$ Department of Gastroenterology, University Hospital, CHU Liège, Belgium

${ }^{\mathrm{b}}$ Department of Biostatistics, University Hospital, CHU Liège, Belgium

\section{A R T I C L E I N F O}

\section{Article history:}

Received 21 August 2020

Accepted 31 October 2020

Available online $\mathrm{xxx}$

\section{Keywords:}

Biologics

Crohn's disease

Inflammatory bowel disease

Real-life data

Ustekinumab

\begin{abstract}
A B S T R A C T
Background: The pivotal clinical trials have largely demonstrated the efficacy and safety of ustekinumab in Crohn's disease. Real-life cohorts published so far only include very few bio-naïve patients. This study assesses effectiveness and safety of ustekinumab in bio-naïve and bio-failure patients treated with ustekinumab in routine practice and look for predictors of response.

Methods: We performed a retrospective monocentric study. Initial response was assessed by maintenance therapy beyond week 16 . Sustained response was assessed by the continuation or cessation of therapy over time for another reason than stopping in sustained remission. Treatment persistence was assessed by Kaplan Meier curves and predictors of treatment persistence were studied by univariate and multivariate Cox model.

Results: Out of 156 recorded patients, three patients were still in their induction phase at time of analysis and 5 patients were lost to follow-up, leaving 148 patients for clinical effectiveness analyses, including 35 bio-naïve when starting ustekinumab. A maintenance therapy was initiated in $79.7 \%$. At one year, the probability to be still treated with ustekinumab was $73.8 \%$. Treatment cessation increased with smoking in multivariate analysis. Previous biologic failure (as a whole), CRP and fecal calprotectin baseline levels did not influence initial response and treatment persistence.

Conclusion: A large proportion of $\mathrm{CD}$ patients initially respond to ustekinumab and continue this treatment beyond one year. Treatment persistence is as high in bio-failure as in bio-naïve patients.
\end{abstract}

(c) 2020 Editrice Gastroenterologica Italiana S.r.l. Published by Elsevier Ltd. All rights reserved.

\section{Introduction}

Crohn's disease (CD) is a chronic inflammatory condition of the gastrointestinal tract whose incidence is increasing worldwide. The symptoms are variable depending on disease location, disease behavior and activity. CD evolves by alternating periods of relapse and remission. This chronic pathology can lead to serious complications that require hospitalization, surgery and lead to disability. The patients present with high disease burden, poor quality of life and they often need treatment throughout life [1,2].

The last approved drug for $C D$ is ustekinumab. Ustekinumab is a monoclonal immunoglobulin produced by the ADN recombinant technology. This agent has been designed to fix two cytokines: interleukin (IL)-12 and IL-23. By blocking these interleukins, ustekinumab acts on T-cells, natural killer cells, antigen-presenting cells and on both Th1 and Th17 cells [3]. It decreases the inflammatory

\footnotetext{
* Corresponding author.

E-mail address: edouard.louis@uliege.be (E. Louis).
}

immune response and the symptoms of $\mathrm{CD}$. Ustekinumab was initially approved for the treatment of psoriasis. In 2016 it was approved in Europe for the treatment of moderate to severe $\mathrm{CD}[4,5]$.

The pivotal clinical trials have largely demonstrated the efficacy and safety of ustekinumab for patients who were refractory to other biologic treatments but also for biologic-naïve patients [6-8]. A large number of real-life experience studies across western world have been published so far, confirming efficacy and safety of ustekinumab in routine practice [9-12]. No clear predictive factor of response has emerged so far, but almost all the patients included in those published real-life cohorts had previously failed one or several other biologics [13-19] . Therefore, it is important to continue to collect real-life data not only in biofailure but also in bio-naïve patients, to compare response-rate and safety in these two populations and to look for predictive factors of response.

The aim of our study was to assess short or long- term response to ustekinumab as well as safety in routine practice in both bionaïve and bio-failure $C D$ and to look for predictors of response. 


\section{Methods}

\subsection{Study design and selection of patients}

A retrospective monocentric study was performed in the department of gastroenterology of the University Hospital of Liège. All patients who started a treatment with ustekinumab in our department between October 2016 and May 2020 were included in the study. Inclusion criteria were: age $\geq 18$ years, diagnosis of moderate to severe $C D$ (moderate to severe $C D$ is a prerequisite for ustekinumab reimbursement in Belgium and is based on the general judgement of the clinician; ustekinumab can be used in bio-naïve patients or in bio-failure patients), initiation of ustekinumab therapy during the study period. All the patients first received an infusion with a weight-based dose of ustekinumab, and then were treated with $90 \mathrm{mg}$ ustekinumab subcutaneously every 8 weeks. Every patient who received at least the intravenous dose was included in the study.

The study was approved by the local ethics committee. Every medical record was analyzed to build an anonymized data base.

\subsection{Outcomes definition}

The main objective was to assess effectiveness of ustekinumab in bio-naïve and bio-failure patients in routine practice. Other major objectives were to analyze the evolution of biomarkers during treatment to look for predictors of response and to analyze the safety of this therapy.

Assessment of effectiveness was restricted to patients having reached week 16 after induction at the time of the analysis. Absence of initial response was defined by the absence of maintenance therapy beyond week 16 . Absence of sustained response was defined by the cessation of therapy for another reason than stopping in sustained remission. C-reactive protein (CRP) and fecal calprotectin ( $\mathrm{FC}$ ) were measured in the majority of the patients before starting therapy and then after induction (week 8-24), during maintenance therapy (around 1 year) and at last follow-up visit. A full biologic response was defined by a c-reactive protein (CRP) $<5 \mathrm{mg} / \mathrm{l}$ and a fecal calprotectin (FC) $<250 \mathrm{microg} / \mathrm{g}$.

Parameters analyzed as predictors of the initial and sustained response were: gender, age at diagnosis, duration of disease, smoking, abdominal pain and stool frequency at baseline, stoma, Montreal classification items, perianal disease, extra-intestinal manifestations, mesalazine use, steroid use and weaning, immunosuppressant use, previous use of anti-TNF, previous use of vedolizumab, previous surgery, body mass index, baseline CRP and FC level.

Adverse events appearing during treatment were retrieved from patient's medical notes and described to evaluate the safety profile of ustekinumab in routine practice.

\subsection{Statistics}

Descriptive statistics including demographic data and baseline disease characteristics were generated for all patients who had been treated by ustekinumab. Factors associated with the initiation of maintenance therapy were studied by univariate and multivariate logistic regression. Treatment persistence was assessed by Kaplan Meier curves and predictors of treatment persistence were studied by univariate and multivariate Cox model (described by odds ratio (OR) and $95 \%$ confidence interval $(95 \% \mathrm{CI})$.

Statistical analysis was performed on a SAS program; p-value < 0.05 were considered to be statistically significant.

A minimum sample size was calculated to support the validity of the present data. Considering a treatment persistence at one year of $50 \%$ in the bio-failure patients and $80 \%$ in the bio-naïve pa-

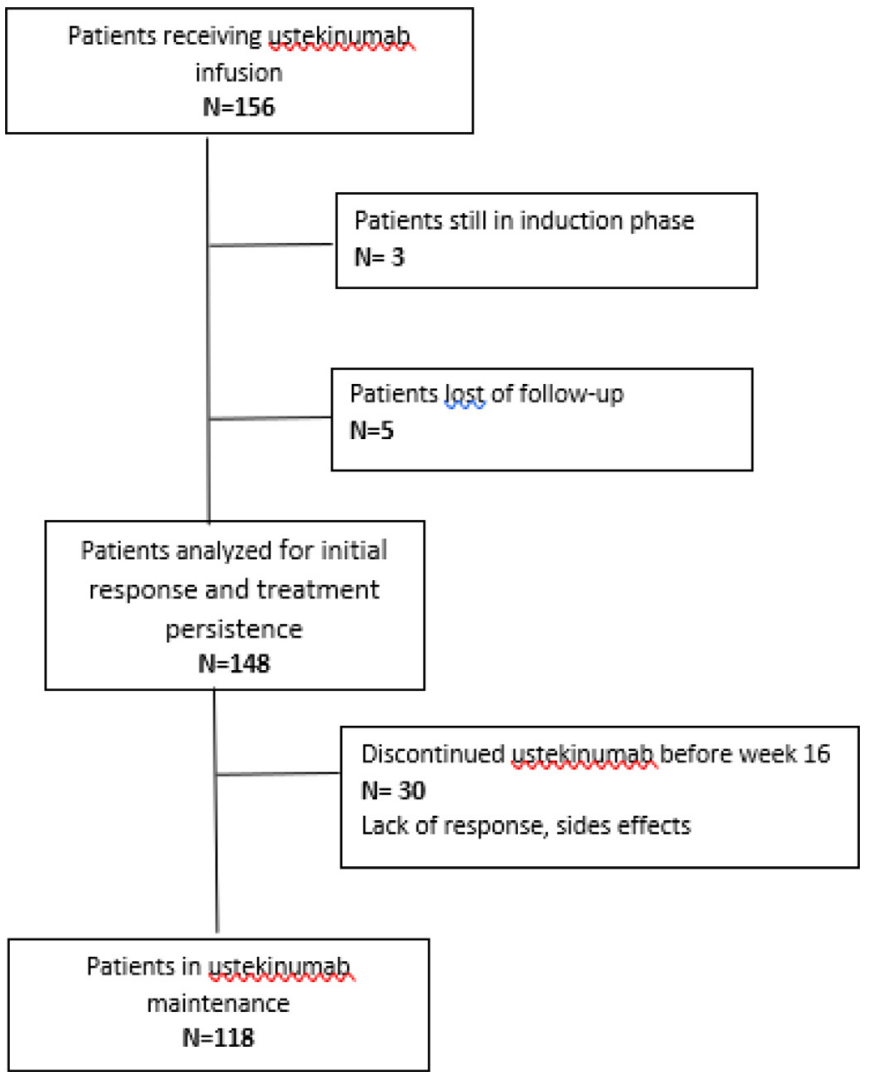

Fig. 1. Flow chart of ustekinumab treatment in the monocentric cohort of $\mathrm{CHU}$ Liège.

tients with a power of $80 \%$ we would have needed 45 patients in each group.

\section{Results}

\subsection{Population description}

After reviewing our database, 156 patients previously refractory to steroids or immunomodulators, who received at least the first weight-based dose ustekinumab infusion were identified. Three (1.9\%) patients were still in their induction phase at the time of analysis and 5 (3.2\%) patients were lost to follow-up. For these reasons, the effectiveness analyses were performed on 148 patients (Fig. 1).

Patients' characteristics are showed in supplementary material (supplementary Table 1) and characteristics for the analyzed cohort in Table 1. Baseline characteristics off bio-naïve and bio-failure patients are compared in Table 2.

\subsection{Initial response}

30 out of 148 patients (20.3\%) did not receive ustekinumab maintenance beyond week 16 .

In univariate analysis, the initiation of maintenance therapy (initial response) showed only association borderline for significance with a series of parameters: less frequent with increasing number of stool/day before treatment (OR 0.93, 95\%CI $0.85-1.01$, $p=0.08$ ), less frequent with abdominal pain (OR 0.26 , 95\%CI $0.06-$ $1.18, p=0.08$ ) less frequent with lower CRP decrease after induction (OR $0.62,95 \% \mathrm{CI} 0.39-1.00, p=0.05$ ). There was no significant impact of other parameters, including being bio-failure or bionaïve, baseline fecal calprotectin or baseline CRP level.

In multivariate analysis, no parameter was significant. 
Table 1

Table 1
Patients' characteristics of the analyzed cohort $(N=148)$.

\begin{tabular}{|c|c|c|}
\hline \multicolumn{2}{|l|}{ Patients' Characteristics } & $\begin{array}{l}\text { Patients analyzed for } \\
\text { effectiveness } n=148\end{array}$ \\
\hline \multicolumn{2}{|l|}{ Male gender $(\mathrm{n}(\%))$} & $68(45.9)$ \\
\hline \multicolumn{2}{|c|}{ Age at the diagnosis (years, mean $\pm S D$ ) } & $28.6 \pm 13$ \\
\hline \multicolumn{2}{|c|}{ Time with illness (years, mean \pm SD)) } & $16 \pm 11.6$ \\
\hline \multirow[t]{2}{*}{ Smoking (n(\%)) } & Yes & $56(42.4)$ \\
\hline & No & $76(57.6)$ \\
\hline \multirow{10}{*}{$\begin{array}{l}\text { Montreal classification (before } \\
\text { ustekinumab) }(n(\%))\end{array}$} & B1 & $102(68.9)$ \\
\hline & B2 & $32(21.6)$ \\
\hline & B3 & $14(9.5)$ \\
\hline & L1 & $50(33.8)$ \\
\hline & $\mathrm{L} 2$ & $25(16.9)$ \\
\hline & L3 & $67(45.3)$ \\
\hline & L4 & $6(4.1)$ \\
\hline & $\mathrm{p}$ (perianal disease): & \\
\hline & Yes & $25(16.9)$ \\
\hline & No & $123(83.1)$ \\
\hline \multirow[t]{2}{*}{ Previous surgery $(\mathrm{n}(\%))$} & Yes & $70(47.6)$ \\
\hline & No & $77(52.4)$ \\
\hline \multirow[t]{3}{*}{ Previous treatment $(\mathrm{n}(\%))$} & Immunomodulator & $111(75.5)$ \\
\hline & Anti-TNF & $110(74.3)$ \\
\hline & Anti-Integrin (vedolizumab) & $59(39.9)$ \\
\hline \multirow[t]{2}{*}{ Concomitant treatment $(\mathrm{n}(\%))$} & IS & $20(13.5)$ \\
\hline & Steroid & $51(34.5)$ \\
\hline \multicolumn{2}{|c|}{$\mathrm{CRP}$ at baseline $(\mathrm{mg} / \mathrm{L}$, mean $\pm \mathrm{SD}),(N=142)$} & $14.8 \pm 23.9$ \\
\hline \multicolumn{2}{|c|}{ FC at baseline $(\mu \mathrm{g} / \mathrm{g}$, mean $\pm \mathrm{SD}),(N=89)$} & $659.6 \pm 903$ \\
\hline
\end{tabular}

Table 2

Comparison of bio-naïve and bio-failure patients among the patients assessed for initial treatment response and treatment persistence $(n=148)$.

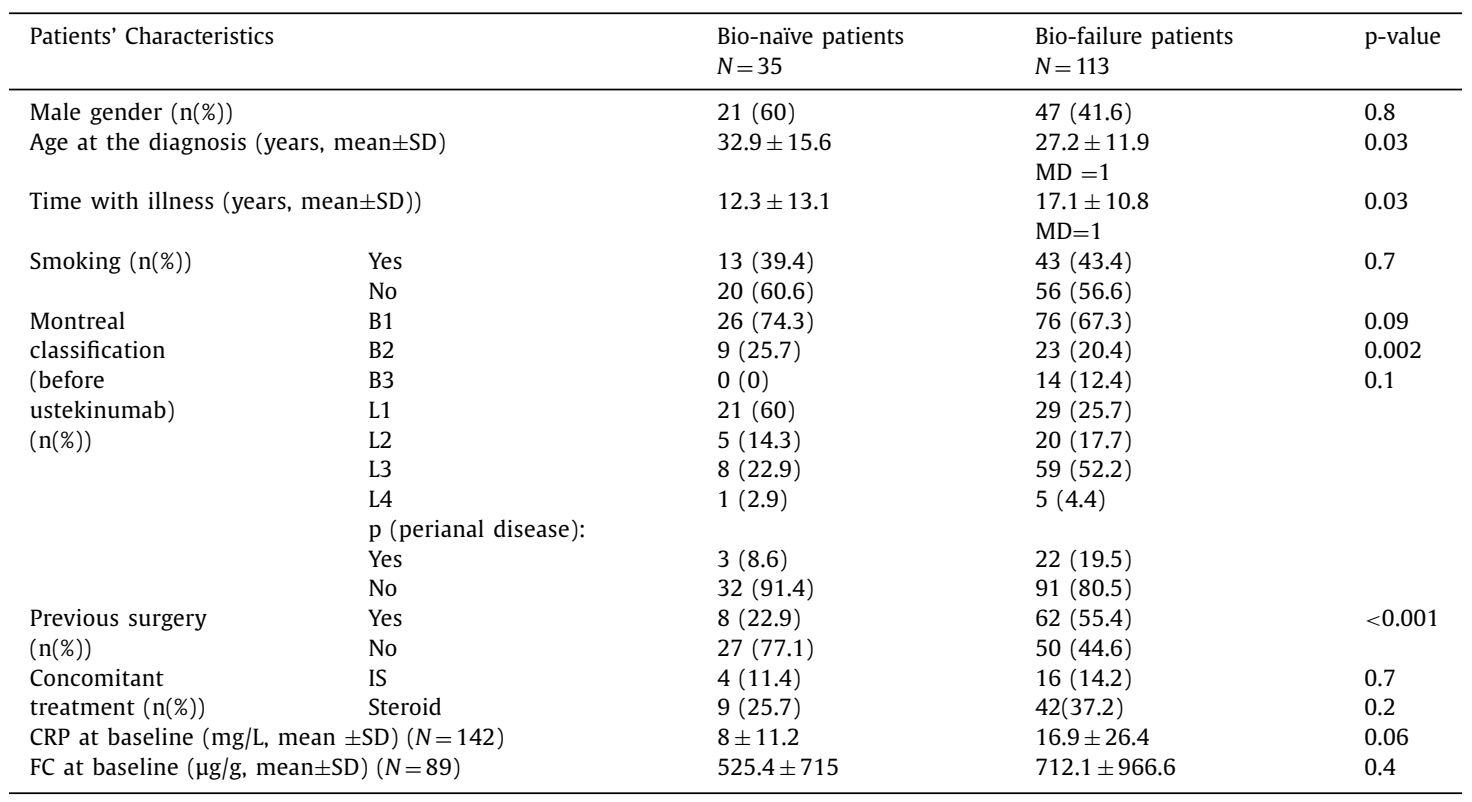

\subsection{Sustained response}

From the 118 patients who continued the treatment after the induction phase, 17 (14.4\%) discontinued the treatment during the maintenance therapy over a median follow-up of 14.9 months. Five of them stopped ustekinumab before one year. All patients started maintenance with a subcutaneous dose of $90 \mathrm{mg}$ every 8 weeks. Seventeen (12.9\%) required an intensification of ustekinumab every 4 weeks. This was significantly more frequent in bio-failure $(16 / 87,18.4 \%)$ than in bio-naïve patients $(1 / 31,3.2 \%)(p=<0.001)$. Only 2 patients decreased the dose to every 12 weeks, both were bio-naïve patients.

Treatment persistence assessed by Kaplan Meier curve is illustrated in Fig. 2. The probability to continue ustekinumab was $82.3 \%$ at 6 months, $73.8 \%$ at one year and $54.4 \%$ at 2 year.
The number of patients starting ustekinumab with steroids was 51 ; the steroid status at week 24 is known in 40 of those who continued a maintenance therapy with ustekinumab. In the bio-failure groups $15 / 32$ (46.9\%) could be weaned from steroids and in the bio-naïve group it was $6 / 8(75 \%)$. There was no difference between the two groups $(p=0.67) .8 / 20$ patients could stop their immunosuppressant and this was more frequent for bio-naïve patients (4/4 bio-naïve patients stopped their immunosuppressant) $(p=0.006)$.

In univariate analysis, treatment cessation increased with active smoking (HR 2.20, CI95\% 1.18-4.11, $p=0.01$ ), the number of stools/day at baseline (HR 1.06, CI95\% 1.00-1.13, $p=0.05$ ), previous exposure to vedolizumab (HR 1.96, CI95\% 1.08-3.56, $p=0.03$ ) and in case of a lower decrease in CRP between baseline and postinduction (HR 1.38, CI95\% 1.02-1.86, $p=0.04$ ). We observed a trend towards a decrease in case of steroid weaning (HR $0.28, \mathrm{CI} 95 \%$ 
A.
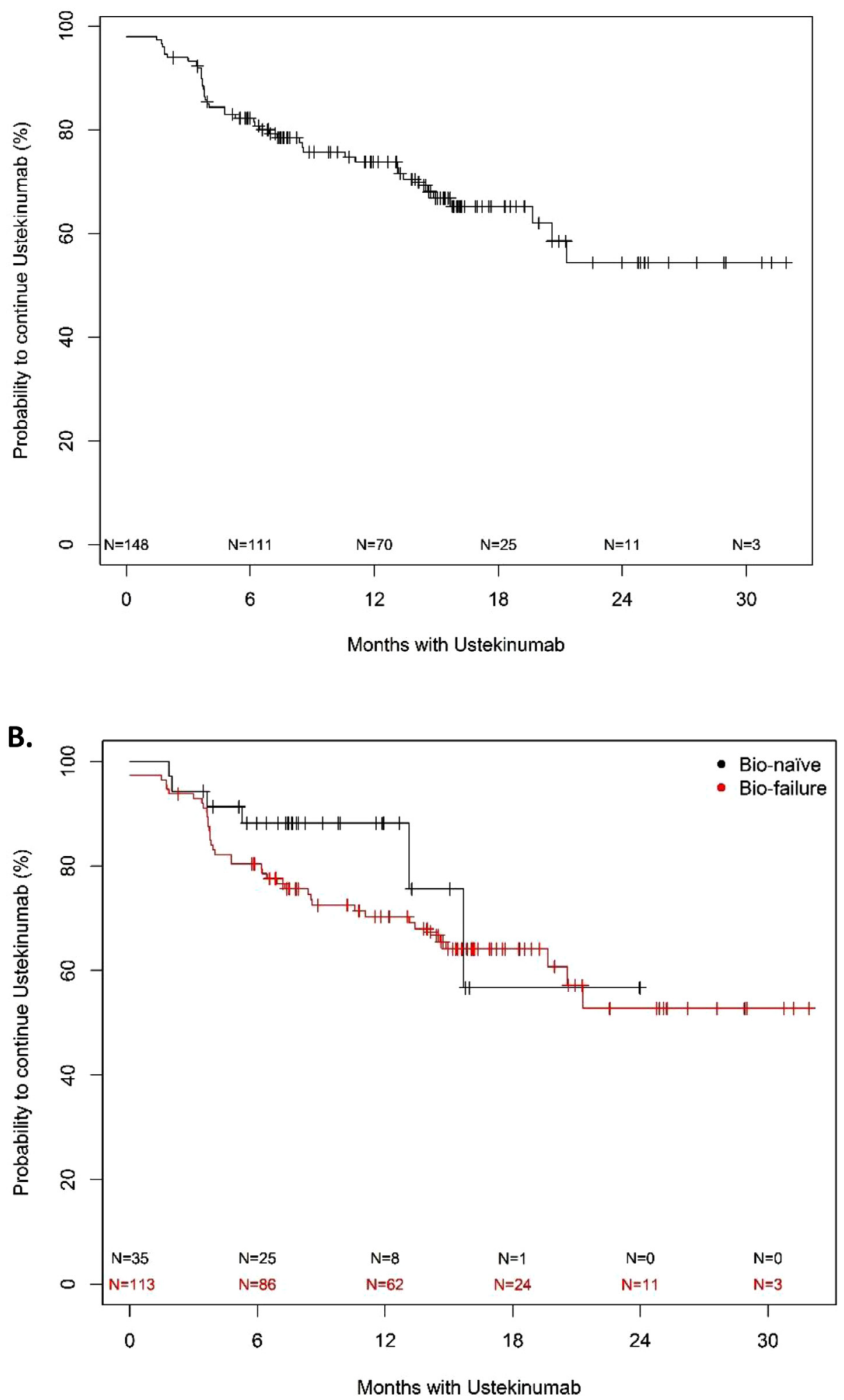

Fig. 2. Probability to continue the treatment by ustekinumab, Kaplan Meier curve. A. for the whole cohort and B. comparing bio-naïve and bio-failure patients.

$0.07-1.05, p=0.06$ ) and with a trend towards an increase in case of previous exposure to an experimental treatment (HR 2.41, CI95\% $0.98-5.93, p=0.06$ ) and abdominal pain (HR 2.47, CI95\% 0.88-6.96, $p=0.09$ ).

In multivariate analysis, only active smoking remained significant (HR 3.1, CI95\% 1.5-6.7, $p=0.003$ ).

\subsection{Evolution of biologic markers}

Figs. 3 and 4, illustrate the evolution of CRP and FC, respectively. The decrease observed after induction was statistically significant both in bio-naïve and bio-failure patients $(p<0.0001)$ and the values were significantly lower in bio-naïve patients $(p=0.03)$. 


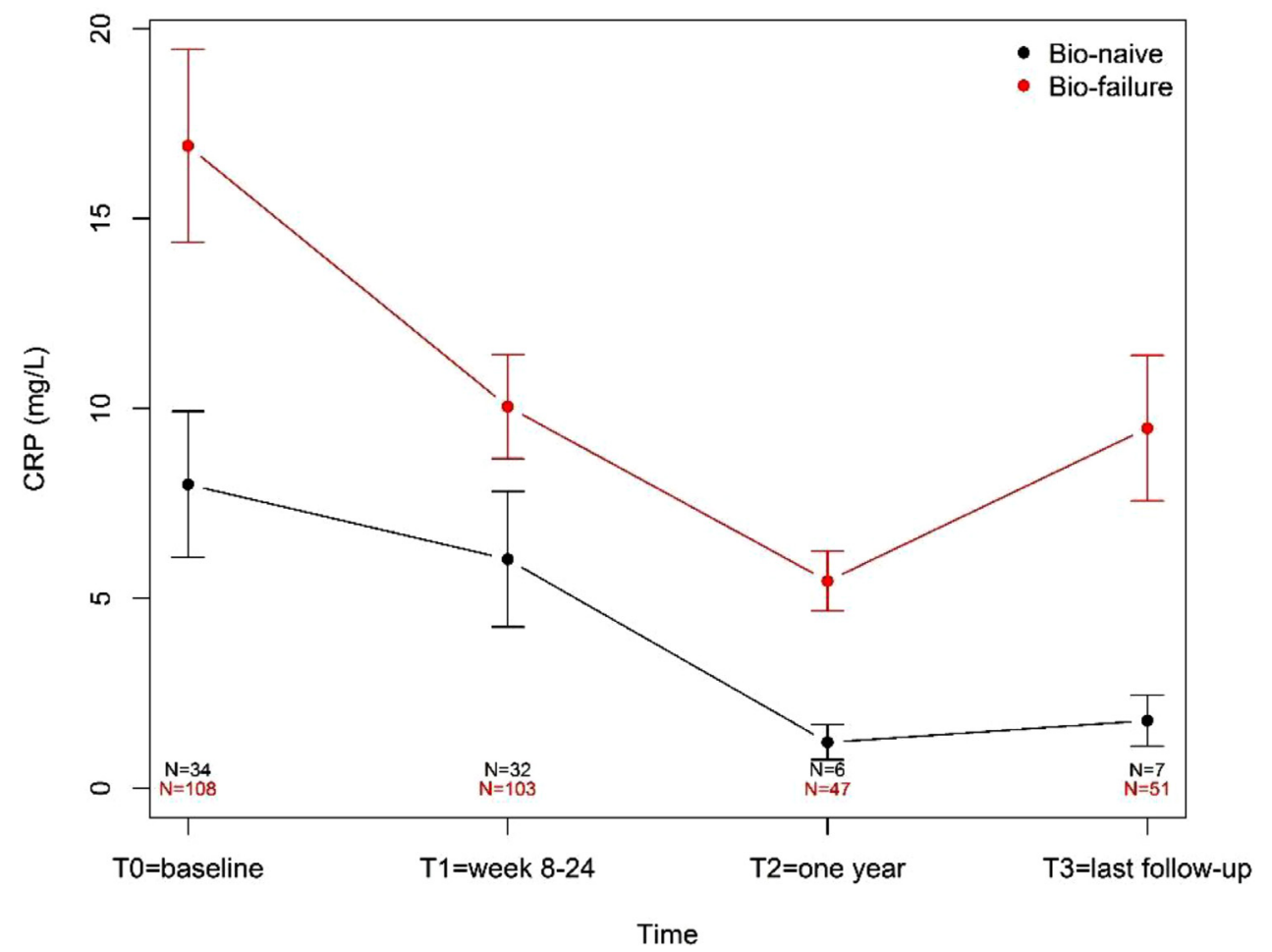

Fig. 3. C Reactive Protein's evolution under ustekinumab.

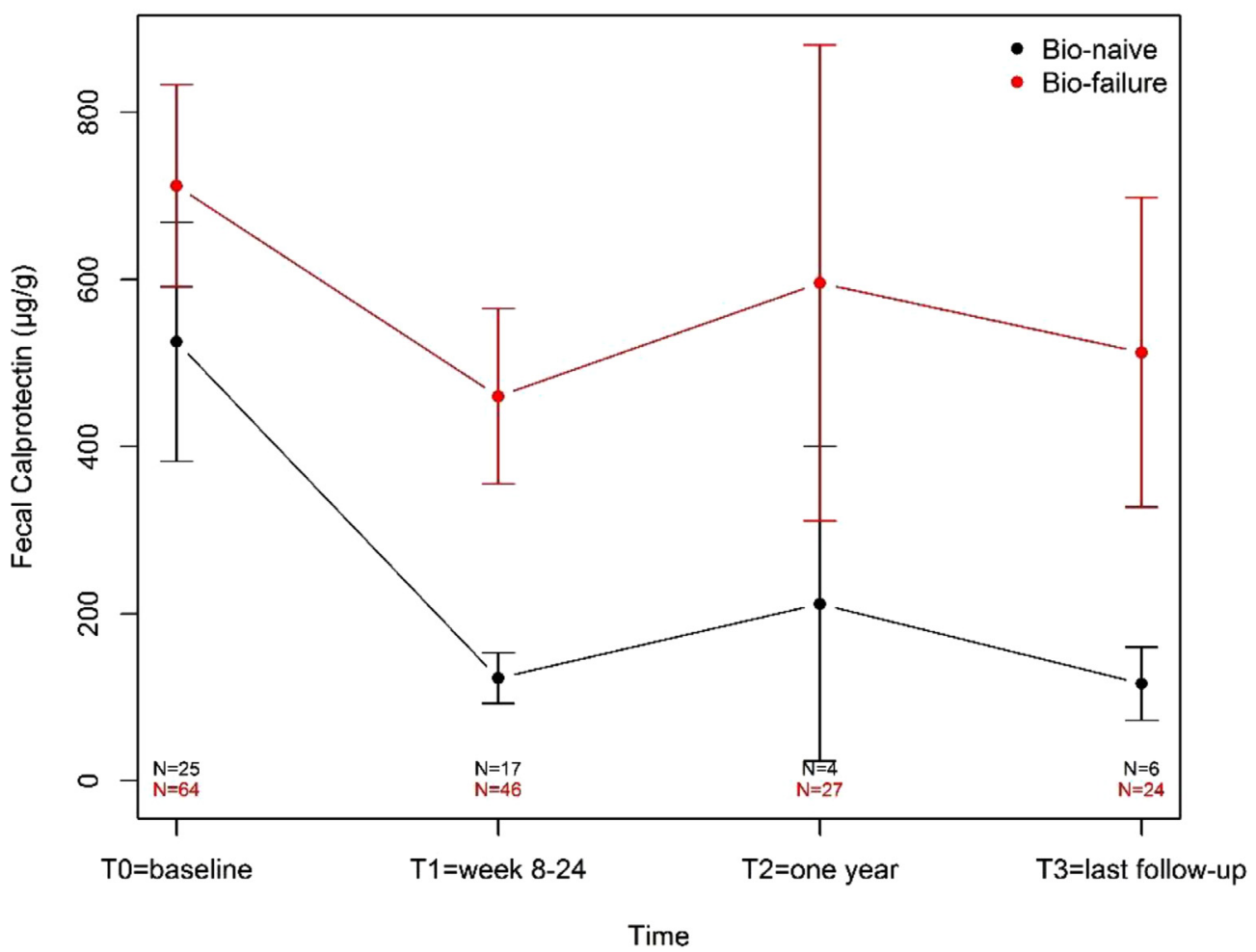

Fig. 4. Faecal calprotectin's evolution under ustekinumab.

Likewise there was a significant decrease in FC over time in both groups $(p<0.0001)$ but not significant difference between bio-naïve and bio-failure patients.

The proportion of patients in full biologic response (CRP < $5 \mathrm{mg} / \mathrm{L}$ and/or $\mathrm{FC}<250 \mu \mathrm{g} / \mathrm{g}$ ) was $62 / 137$ (45.3\%), 37/58 (63.8\%) and 33/63 (52.4\%) after induction, at one year and at last followup, respectively. Proportion of patients with full biologic response was higher in bio-naïve than bio-failure after induction, at one year and last follow-up and reached the statistical significance for this last time point. These results are showed in Table 3.

\subsection{Safety profile}

The safety profile was assessed in the whole population of 156 patients. Hospitalization related to $\mathrm{CD}$ was observed for 27 patients 
Table 3

The proportion of patients in full biologic response, bio-naïve vs bio-failure.

\begin{tabular}{llll}
\hline Time point & Bio-naïve & Bio-failure & p-value \\
\hline After induction, $N=137$ & $20 / 33(60.6 \%)$ & $42 / 104(40.4 \%)$ & 0.05 \\
At one year, $N=58$ & $6 / 7(85.7 \%)$ & $31 / 51(60.8 \%)$ & 0.4 \\
At last follow-up, $N=63$ & $8 / 9(87.5 \%)$ & $25 / 54(46.3 \%)$ & 0.03 \\
\hline
\end{tabular}

(17.8\%). Only $3(2 \%)$ new perianal disease were observed and 7 (4.6\%) patients needed endoscopic dilatation for obstructive stricture. During the treatment period, 6 patients (3.9\%) required intestinal surgical resection.

Infection requiring antibiotics were observed in 26 (17.1\%) cases and infection requiring hospitalization were reported in 7 (4.6\%) patients. The infection requiring antibiotics affected the digestive tract for $12(46.2 \%)$ patients, the respiratory tract for 10 (38.5\%), were cutaneous in 1 (3.8\%), dental infections in $2(7.7 \%)$ cases and concerned urinary tract infection in 1 (3.8\%) case. We observed no opportunistic infection.

The adverse event led to an interruption of ustekinumab in nine cases. For five patients it was during the induction phase and four stopped during maintenance therapy. The side effects leading to a treatment discontinuation were: headache (2), pulmonary infection (1), skin lesions (1), arthralgia (1), breast cancer (1), thymic problem (1) and general intolerance not specified (2).

We observed no death during the study period.

\section{Discussion}

Our monocentric real-life cohort of $C D$ patients treated with ustekinumab shows around three quarter of the patients achieving sufficient primary response and tolerance to undergo maintenance therapy and up to two thirds and half of them still being treated after 1 and 2 years, respectively. Our study is one of the first to assess real life clinical outcome with ustekinumab in both bio-naïve and bio-failure $\mathrm{CD}$ patients. We could show similar treatment persistence in these two subgroups, but a trend towards a stronger biologic response in bio-naïve patients. There was no new safety signal.

The clinical outcomes observed in our cohort are similar to the ones previously reported in other real life multicentric or monocentric cohorts. In these published cohorts, almost all patients were refractory to one or more previous biologics $[11,13,14,18,20,21,22]$. Treatment persistence in such refractory cohort, corresponding to early real-life experience with a new drug, may be biased by the fact that there is no further therapeutic option for those patients and that the clinicians and patients may be satisfied with only partial clinical response, in the absence of clear indication for surgery. If the majority of our patients were also in such situation, we also analyzed a substantial number of patients having failed only one biologic or even being bio-naïve. In such patients, we use to apply a treat-to-target strategy, mainly based on clinical and biomarkers evolution and change therapy if clinical and biomarkers targets are not reached. Therefore, the similar treatment persistence observed in our bio-naïve patients may actually reflect a better disease control in those patients. This hypothesis is reinforced when comparing biomarkers evolution: although there was a significant decrease in both CRP and FC during ustekinumab treatment in both bio-naïve and bio-failure groups, the CRP values were significantly lower in bio-naïve patients. Likewise, the proportion of patients in full biologic response (CRP $<5 \mathrm{mg} / \mathrm{l}$ and/or fecal calprotectin $<250 \mathrm{microg} / \mathrm{g}$ ) was numerically greater in bio-naïve patients. Other aspects differentiating bio-naïve from bio-failure patients, also suggesting a more profound control of the disease in bio-naïve patients, were the more frequent withdrawal of immunosuppressant drug and the lower frequency of dose in- tensification at 4 weeks intervals with even some patients stable at every 12 weeks ustekinumab administration.

The absence of striking difference in initial clinical response and treatment persistence observed between bio-naïve and biofailure patients contrasts with what was observed in similar cohorts with vedolizumab where a lower response rate was clearly observed in bio-failure patients [14,23]. Also in contrast with those vedolizumab real life cohorts, we could not show a lower treatment initial response and persistence in patients with baseline elevated CRP or in younger patients. Logically we also observed that patients achieving steroid weaning after induction had a longer treatment persistence [15].

When looking at previously published real-life CD patients cohorts treated with ustekinumab, predictors of response were heterogeneous $[11,13,14,18,20-22]$. In particular, the absence of impact of a previous anti-TNF treatment was not unanimously found. A first explanation to this discrepancy is that all these previous studies didn't include any or only a very limited number of bio-naïve patients. Another explanation may be that some of these early studies did not use an initial weight-based intra-venous induction [15], which may have lowered the response rate, particularly in more severe or refractory patients. Other heterogeneous predictors found in some previous cohorts included ileocolonic disease, previous intestinal resection, male gender, extra-intestinal manifestations, steroid use and concomitant immunomodulator use $[11,14,20]$. Some previous cohorts showed predictors similar to ours, including smoking associated with poorer response to ustekinumab [21]. While in previously published real-life cohorts with vedolizumab in $C D$, predictors of response were rather consistent, highlighting a worse response in patients with previous antiTNF failure and higher disease burden [14,23], this seems to be less clear in real-life ustekinumab $C D$ cohorts like in ours. In the present cohort there was no impact of a previous biologic treatment on the response to ustekinumab, although intriguingly, a previous use of vedolizumab (which most often followed a previous use of anti-TNF) was associated to a lower rate of ustekinumab persistence. This is probably reflecting lower persistence in multirefractory patients but the specific role of previous vedolizumab failure would deserve further analyses. Overall, the comparative response to various biologic treatments in patients failing other biologics is an important point that requires clarification to determine optimal treatment sequences in $\mathrm{CD}$.

We observed no new safety signal. Most frequent adverse events were hospitalizations for Crohn's disease or infections requiring antibiotics. The nature of the side effects and the rate of infection are in line with the UNITI-1, UNITI-2 and IM-UNITI trials. We observed no opportunistic infection and no death. The side effects only rarely led to treatment cessation. These safety outcomes are also similar to other real-life ustekinumab studies [7,11,20,22].

Our study has some limitations. It is a retrospective monocentric study limiting the amount of data to assess initial and sustained response to therapy. For this reason, we focused on robust outcomes: initiation of maintenance therapy for initial response and treatment persistence for sustained response. Because treatment persistence may reflect different clinical outcomes in bio-failure and bio-naïve patients, and because the number of bio-naïve patients available for this analysis was a bit low, we must interpret the comparison between bio-failure and bio-naïve patients with caution. Furthermore, we don't have sufficient data on endoscopic healing. In our practice, we tend to favor biomarkers, including FC and CRP to apply a treat-to-target strategy. These objective markers could confirm the favorable clinical evolution observed in the majority of our patients. Beside these limitations, to our knowledge, our real-life study included the largest number of bio-naïve patients so far, which allowed us to show, for the first time, a comparable treatment persistence in bio-failure and 
bio-naïve patients but to also suggest a more profound biologic response in bio-naïve patients.

In conclusion, Ustekinumab seems to provide sustained benefit in a substantial proportion of $C D$ patients in real-life practice, whatever the previous biologic treatment status and whatever the initial inflammatory burden.

\section{Declaration of Competing Interest}

E Louis has received fees for: Research Grant: Takeda, Pfizer; Educational Grant: Abbvie, Takeda, Janssen; Speaker Fees: Abbvie, Ferring, MSD, Falk, Takeda, Hospira, Janssen, Pfizer, Celgene; Advisory Board: Abbvie, Ferring, MSD, Takeda, Celgene, Hospira, Janssen; Consultant: Abbvie.

\section{Supplementary materials}

Supplementary material associated with this article can be found, in the online version, at doi:10.1016/j.dld.2020.10.040.

\section{References}

[1] Zaltman C, Amarante H, Machado MB, et al. Crohn's disease - treatment with biological medication. Revista da Associação Médica Brasileira 2019:65:554-67.

[2] Na S-Y, Moon W. Perspectives on current and novel treatments for inflammatory bowel disease. Gut Liver 2019;13:604-16.

[3] Ma C, Panaccione R, Khanna R, et al. IL12/23 or selective IL23 inhibition for the management of moderate-to-severe Crohn's disease? Best Pract Res Clin Gastroenterol. 2019:38-9.

[4] Engel T, Kopylov U. Ustekinumab in Crohn's disease: evidence to date and place in therapy. Ther Adv Chronic Dis 2016:7:208-14.

[5] Khanna R, Preiss JC, MacDonald JK, et al. Anti-IL-12/23p40 antibodies for induction of remission in Crohn's disease. Cochrane Database Syst Rev 2015:5.

[6] Li K, Friedman JR, Chan D, et al. Effects of Ustekinumab on histologic disease activity in patients with Crohn's disease. Gastroenterology 2019;157:1019-31.

[7] Feagan BG, Sandborn WJ, Gasink C, et al. UNITI-IM-UNITI study group. Ustekinumab as induction and maintenance therapy for Crohn's disease. N Engl J Med 2016:375:1946-60.

[8] Eberl A, Hallinen T, Af Björkesten C-G, et al. Ustekinumab for Crohn's disease: a nationwide real-life cohort study from Finland (FINUSTE). Scand J Gastroenterol 2019;54:718-25.
[9] Biemans VBC, Van der Meulen-de Jong AE, Van der Woude CJ, et al. Ustekinumab for Crohn's disease: results of the ICC Registry, a nationwide prospective observational cohort study. J Crohns Colitis 2019.

[10] Khorrami S, Ginard D, Marín-Jiménez I, et al. Ustekinumab for the treatment of refractory Crohn's disease: the Spanish experience in a large multicentre open-label cohort. Inflamm Bowel Dis 2016;22:1662-9.

[11] Liefferinckx C, Verstockt B, Gils A, et al. Long-term clinical effectiveness of ustekinumab in patients with Crohn's disease who failed biologic therapies: a National Cohort Study. J Crohns Colitis 2019;13:1401-9.

[12] Kopylov U, Afif W, Cohen A, et al. Subcutaneous ustekinumab for the treatment of anti-TNF resistant Crohn's disease-the McGill experience. J Crohns Colitis 2014;8:1516-22.

[13] Greenup A-J, Rosenfeld G, Bressler B. Ustekinumab use in Crohn's disease: a Canadian tertiary care centre experience. Scand J Gastroenterol 2017;52:1354-9.

[14] Barré A, Colombel J-F, Ungaro R. Review article: predictors of response to vedolizumab and ustekinumab in inflammatory bowel disease. Aliment Pharmacol Ther 2018;47:896-905.

[15] Wils P, Bouhnik Y, Michetti P, et al. Long-term efficacy and safety of ustekinumab in 122 refractory Crohn's disease patients: a multicentre experience. Aliment Pharmacol Ther 2018;47:588-95.

[16] Sandborn WJ, Rutgeerts P, Gasink C, et al. Long-term efficacy and safety of ustekinumab for Crohn's disease through the second year of therapy. Aliment Pharmacol Ther 2018;48:65-77.

[17] Ahmed Z, Venkata K, Zhang N, et al. Comparative effectiveness of ustekinumab versus adalimumab in induction of clinical response and remission in Crohn's disease: experience of a real-world cohort at a tertiary care inflammatory bowel disease referral center. Gastroenterology Res 2019;12:245-51.

[18] Battat R, Kopylov U, Bessissow T, et al. Association between ustekinumab trough concentrations and clinical, biomarker, and endoscopic outcomes in patients with Crohn's Disease. Clin Gastroenterol Hepatol 2017:15:1427-34 e2.

[19] Ma C, Fedorak RN, Kaplan GG, et al. Long-term maintenance of clinical, endoscopic, and radiographic response to ustekinumab in moderate-to-severe Crohn's disease: real-world experience from a multicenter cohort study. Inflamm Bowel Dis 2017;23:833-9.

[20] Hoffmann P, Krisam J, Wehling C, et al. Ustekinumab: "Real-world" outcomes and potential predictors of nonresponse in treatment-refractory Crohn's disease. World J Gastroenterol 2019;25:4481-92.

[21] Casas Deza D, García López S, Lafuente Blasco M, et al. Efficacy and safety of ustekinumab in real clinical practice. Retrospective multicentre study. ARAINF cohort. Gastroenterol Hepatol. 2019.

[22] Kubesch A, Rueter L, Farrag K, et al. Short and Long-term effectiveness of ustekinumab in patients with crohn's disease: real-world data from a German IBD cohort. J Clin Med 2019:8.

[23] Dulai PS, Boland BS, Singh S, et al. Development and validation of a scoring system to predict outcomes of vedolizumab treatment in patients with Crohn's disease. Gastroenterology 2018;155:687-95 .e10. 